\title{
Interaction between intra-grain currents and transport current in bulk $\mathrm{YBaCuO}^{*}$
}

\author{
A.R. Kuper, H. Hemmes and H. Rogalla \\ University of Twente, Department of Applied Physics, PO Box 217, NL-7500 AE \\ Enschede, The Netherlands
}

\begin{abstract}
The hysteric magnetization curves of transport and grain currents are measured for a ring shaped sample by applying an a.c. magnetic field and using pickup coils. The dependence of the magnetization curves on the amplitude of the applied a.c. magnetic field can be explained by a simple model. This model takes into account the time dependence of the transport and the intra-grain current. We will show that the mutual interaction of the grain and transport currents cannot be neglected in our samples. At first the influence of an additional d.c. background field on the transport current seems quite unexpected, but this d.c. field dependence can be interpreted too within our model.
\end{abstract}

Keywords: bulk YBaCuO; magnetization; inter- and intra-grain currents

Magnetization measurement techniques to determine the critical current density $J_{c}$ of ceramic superconductors are widely used ${ }^{1-5}$. However, the reliability of the obtained $J_{c}$ values is limited by the model used to extract these values from experimental data. Usually Bean's critical state model is applied with some magnetic induction dependent critical current density $J_{\mathrm{c}}(B)^{6.7}$. Bean's model assumes the same $J_{\mathrm{c}}(B)$ throughout the sample. This model generally cannot apply for granular bulk sintered high $\mathbb{T}_{\mathrm{c}}$ materials with very heterogeneous properties. Because of their granular character, the magnetization of bulk sintered high $T_{\mathrm{c}}$ superconductors is thought to consist of two contributions. These originate from inter-grain and intra-grain currents. The inter-grain current is usually associated with the transport current. In solid samples the magnetization signal of the intra-grain currents is dominant ${ }^{4}$. Using ring shaped samples therefore has two advantages. First, the transport current contribution to the magnetization signal is enhanced with respect to the grain current contribution. This is because the enclosed area of the current loop is much larger than in a solid sample. Second, the ring geometry makes separation of the two current contributions possible ${ }^{8}$. The enhancement of the magnetization related to the transport current and the separation of the transport and grain current contributions facilitates interpretation of the experimental data with respect to conventional magnetization techniques.

\footnotetext{
*Paper presented at the conference 'Critical Currents in High $T_{c}$ Superconductors', $22-24$ April 1992, Vienna, Austria

$0011-2275 / 92 / 111027-07$

(C) 1992 Butterworth - Heinemann Ltd
}

\section{Experimental technique}

Sintered bulk high $T_{\mathrm{c}}$ superconductors consist of grains with a 'high' $J_{c}$ connected by weak links with a 'low' $J_{c}{ }^{\prime}$. In ring shaped samples an applied a.c. magnetic field induces currents. The current loop closed around the ring core (loop 1, Figure la) is associated with the transport current as determined by resistance measurements. Because of the low inter-grain $J_{c}$, the magnetic shielding of the ring is incomplete. As a result shielding currents will flow inside the grains (loop 2, Figure la).

The magnetic induction inside and outside a closed current loop points in opposite directions (see Figure $l b$ ). Therefore, inside the ring the magnetic induction generated by the transport and grain currents has opposite signs. Outside the ring the senses are the same. This difference is used to separate the magnetization originating from the transport and grain currents. The magnetic flux is measured with a single pickup coil in the ring and a double pickup coil surrounding the ring. The induced pickup voltages $U_{i(0)}$ inside and outside the ring are given by

$U_{\mathrm{i}}=-M_{\mathrm{it}} \dot{I}_{\mathrm{t}}-g_{\mathrm{ig}} \dot{M}_{\mathrm{g}}$

$U_{\mathrm{o}}=-M_{\mathrm{ol}} \dot{I}_{\mathrm{t}}-g_{\mathrm{og}} \dot{M}_{\mathrm{g}}$

where $M_{\mathrm{i}(0) \text { it }}$ is the mutual inductance of the transport current with the pickup set inside (outside) the ring, $I_{\text {, }}$ is the time derivative of the transport current and $g_{\text {itong }}$ is the coupling factor of the time derivative of the average grain magnetization $\dot{M}_{\mathrm{g}}$ with the inner (outer) pickup set. The signal arising from the coupling with the field coil is not included in Equation (1) as it is assumed to be eliminated by compensating coils. $M_{\mathrm{i}(\mathrm{o}) \mathrm{t}}$ and $g_{\mathrm{i}(\mathrm{o}) \mathrm{g}}$ 


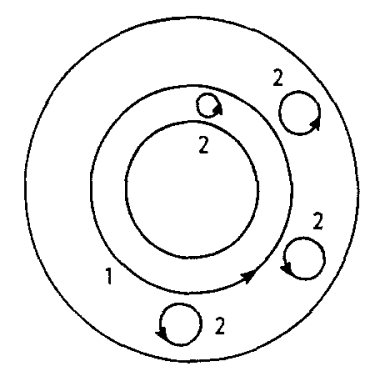

a

Figure 1 (a) The two types of current loops in the ring. Loop 1 is the transport current and loop 2 the intra-grain current; (b) the senses of the magnetic induction inside $B_{i}$ and outside $B_{0}$ a closed current loop with current $l_{\text {r }}$

are constant if we assume a fixed current and magnetization distribution.

The sign of the mutual inductances $M_{i(o) t}$ and the coupling factors $g_{\text {i(o)g }}$ depends on the sense of the flux measured. Using the sign convention of Figure $1 b$, we find

$M_{\text {it }}>0$

$g_{\mathrm{og}}, g_{\mathrm{ig}}$ and $M_{\mathrm{ot}}<0$

Solving Equations (1a) and (1b) for the transport current related component $U_{\mathrm{t}}$ and the grain magnetization related component $U_{\mathrm{g}}$ yields

$U_{\mathrm{t}} \equiv U_{\mathrm{o}}-\beta_{\mathrm{t}} U_{\mathrm{i}}=-\left(M_{\mathrm{it}}-\beta_{\mathrm{t}} M_{\mathrm{ot}}\right) \dot{I}_{\mathrm{t}}$

$U_{\mathrm{g}} \equiv U_{\mathrm{o}}-\beta_{\mathrm{g}} U_{\mathrm{i}}=-\left(g_{\mathrm{og}}-\beta_{\mathrm{g}} g_{\mathrm{ig}}\right) M_{\mathrm{g}}$

The weight factor $\beta_{t(g)}$ is defined by

$\beta_{\mathrm{t}}=g_{\text {og }} / g_{\text {ig }}$

$\beta_{\mathrm{g}}=M_{\mathrm{it}} / M_{\mathrm{ot}}$

As $\beta_{1}>0$ and $\beta_{\mathrm{g}}<0$ Equation (3a) corresponds to subtraction and Equation (3b) to addition of the inner and outer pickup coil signals. That is, by subtracting the signals of the pickup coils outside and inside the ring with a weight factor $\beta_{\text {t }}$ we get a signal proportional to the time derivative of the transport current (Equation (3a)). Adding those signals with a weight factor $\beta_{\mathrm{g}}$ yields a signal proportional to the time derivative of the average grain magnetization (Equation (3b)). Once the mutual inductances and the coupling factors in Equation (3) are known, the transport current and the average grain magnetization can be calculated by integrating the pickup voltages $U_{t}$ and $U_{\mathrm{g}}$.

Assuming coaxial symmetry and a homogeneous transport current distribution, the formalism of Fawzi and Burke ${ }^{9}$ can be used to calculate the mutual inductances. If we approximate the average grain magnetization by screening currents flowing in opposite direction on the inner and outer surfaces of the ring, the coupling factors $g_{\text {i(o)g }}$ can also be calculated applying the same formalism.

The a.c. field coil, pickup coils and ring are inserted in a flow cryostat, which in turn is placed inside the bore of a $5 \mathrm{~T}$ solenoid. The maximum a.c. field amplitude is limited to about $20 \mathrm{mT}$ by heat dissipation of the a.c. field coil.

The $\mathrm{YBa}_{2} \mathrm{Cu}_{3} \mathrm{O}_{7-\delta}$-ring was pressed from powder made by citrate synthesis and pyrolysis ${ }^{10}$. An organic binder was added to facilitate pressing ${ }^{11}$. After pressing, the ring was sintered in flowing oxygen for $10 \mathrm{~h}$ at $925^{\circ} \mathrm{C}$. The oxygen content was restored by keeping the temperature constant at $450^{\circ} \mathrm{C}$ for $15 \mathrm{~h}$.

\section{Description of the model}

As pointed out in the introduction, bulk ceramic superconductors can have very heterogeneous properties. This conflicts with the assumption of a single $J_{c}(B)$ relation for the interpretation of the magnetization data in terms of a critical state model.

Frankel $^{12}$ and more recently Däumling and Larbalastier $^{13}$ applied a finite element technique to determine $J_{c}$ from the magnetic field of a disc using the critical state model and a static current distribution. Our model extends the finite element technique to the description of the response of the current distribution to a time dependent magnetic field ${ }^{14}$. For this purpose the ring is divided into concentric segments that are inductively coupled to each other and to the a.c. field coil. The time dependent magnetic field induces an electric field in the ring. The induced currents in each segment depend on the local magnitude of the electric field and the (non-linear) resistivity of the segments. Then the time dependent current in a segment $I_{\mathrm{i}}$ is related to the current in the field coil $I_{\mathrm{f}}$ and the currents in all the other segments, as given by

$M_{i \mathrm{i}} \dot{I}_{i}+R_{i} I_{i}=-M_{\mathrm{if}} \dot{I}_{\mathrm{f}}-\sum_{j \neq i} M_{i j} \dot{I}_{j}$

Here $M_{i \mathrm{i}}$ is the self inductance of segment $i, \dot{I}_{i}$ the time derivative of the current in segment $i, R_{i}$ the resistance and $M_{i f(j)}$ the mutual inductance of segment $i$ with the field coil (segment $j$ ). The resistance of the segment is given by $R_{i}=\rho_{i} 2 \pi r_{i} / A_{i}$ where $A_{i}$ is the cross-section of the segment normal to the transport current. For the resistivity $\rho_{i}$ of the segment with radius $r_{i}$ we use the expression

$\rho_{i}= \begin{cases}\rho_{\mathrm{n}}\left(J_{i} / J_{\mathrm{c}}\left(B_{i}\right)\right)^{n} & J_{i} \leq J_{\mathrm{c}}\left(B_{i}\right) \\ \rho_{\mathrm{n}} & J_{i}>J_{\mathrm{c}}\left(B_{i}\right)\end{cases}$

with $\rho_{\mathrm{n}}$ the normal state resistivity and $J_{i}$ the current density $I_{i} / A_{i} . J_{c}\left(B_{i}\right)$ is the magnetic induction dependent critical current density given by the Kim relation?: $J_{c}(B)=J_{0} /\left(1+\left|B / B_{0}\right|\right)$. In this expression $J_{0}$ is the critical current density at zero magnetic induction and $B_{0}$ determines the sensitivity of $J_{\mathrm{c}}$ to $B$.

The grains respond to the local field in the ring. They are magnetized with a magnetization $M_{\mathrm{g}}$. This magnetization generates a field

$B_{\mathrm{g}}=-\alpha \mu_{0} M_{\mathrm{g}}$

The factor $\alpha$ depends on the position relative to the grain ( $\mu_{0}$ is the magnetic permeability of a vacuum). The transport current flows through weak links at grain 
boundaries. We assume its path lies outside the intragrain current loops everywhere. In that case $\alpha$ is positive. The other grains also feel $B_{\mathrm{g}}$ and they lie outside too. So for the other grains $\alpha$ is also positive. Taking the average $M_{\mathrm{g}}$ for a segment, $B_{\mathrm{g}}$ is the same for the transport current and the grains. The local field for the transport current and the grains is then given by

$B_{i}=B_{\mathrm{a}}+\sum_{j \neq i} B_{i j}-\alpha \mu_{0} M_{\mathrm{g} i}$

$B_{\mathrm{a}}$ is the applied magnetic induction and $B_{i j}$ is the magnetic induction at segment $i$ generated by the transport current in segment $j$. As $M_{\mathrm{g} i}$ points in the opposite direction to the applied field its contribution increases the local field. To avoid elaborate calculations the volume of each segment is thought to be entirely filled with spherical grains of the same size. In the unsaturated regime the magnetization is determined by the diamagnetic response. The change in the magnetization $M_{\mathrm{gi}}$ from time $t-\delta t$ to $t$ is proportional to the change in the local magnetic induction $B_{i}$ in that time interval

$\mu_{0} M_{\mathrm{g} i}(t)=\mu_{0} M_{\mathrm{g} i}(t-\delta t)-\gamma\left(B_{i}(t)-B_{i}(t-\delta t)\right)$

Here $\gamma$ is the demagnetization coefficient of the grain. If the magnetization saturates it is given by

$\mu_{0} M_{\mathrm{gi}}(t)=\mu_{0} M_{\mathrm{g}}^{\mathrm{s}}\left(B_{i}^{\mathrm{g}}(t), \operatorname{sign}\left(\dot{B}_{i}^{\mathrm{g}}(t)\right)\right)$

The saturated magnetization is not only dependent on the absolute value of the magnetic induction but also on the sign of its time derivative. In the implementation of the model the diamagnetic response of the grain is calculated first. If the calculated magnetization exceeds the saturation value it is set to the saturation value. As $M_{\mathrm{g}}^{\mathrm{s}}$ depends on the local field and the local field depends on the magnetization, this is an iterative procedure.

\section{Results and discussion}

Measurements were performed on a sintered ring pressed from $\mathrm{YBa}_{2} \mathrm{Cu}_{3} \mathrm{O}_{7-\delta}$ powder with an inner (outer) diameter of 21 (25) $\mathrm{mm}$ and a thickness of $2.2 \mathrm{~mm}$. The amplitude of the applied a.c. field was varied up to $20 \mathrm{mT}$. For an a.c. amplitude of $10 \mathrm{mT}$ the d.c. background field was varied in steps of $10 \mathrm{mT}$. The sample temperature was kept at $70 \mathrm{~K}$.

Measured magnetization curves for different a.c. field amplitudes are shown in Figure 2. For small amplitudes the curve for the magnetization associated with the transport current (Figure $2 a$ ) is similar to the magnetization found for thin films or single crystals ${ }^{15.16}$. At larger amplitudes of the applied magnetic induction $B_{\text {a }}$ the maximum of the transport current shifts towards the $B_{\mathrm{a}}=0$ axis (Figure $2 c$ ). At an amplitude of $20 \mathrm{mT}$ the maximum coincides with that axis (Figure $2 e$ ). The maximum crosses the axis if we increase the amplitude further.

At small amplitudes the grain magnetization is linear with $B_{\mathrm{a}}$, with hysteresis and a plateau around $B_{\mathrm{a}}=0$ (Figures $2 b$ and $2 d$ ). These curves are, except for the plateau, similar to the grain magnetization measured by Calzona et al. $^{+}$, but at larger amplitudes the magnetization starts to saturate (Figure 2f). Hysteresis occurs at an amplitude where the transport current saturates. From that point on, the plateau starts to develop.

For our model calculations we assumed a typical grain diameter of $10 \mu \mathrm{m}$, as found in SEM photographs taken from cross-sections of similar samples. The magnetization of saturated grains was determined by calculation. The current density in the grain was also described with a $\mathrm{Kim}$ relation $\left(J_{c}^{\mathrm{g}}(B)=J_{\delta}^{\mathrm{y}} /\left(1+\left|B / B_{0}^{\mathrm{g}}\right|\right)\right.$. $J_{0}^{\mathrm{y}}$ is three orders of magnitude higher than for the transport current (e.g. Reference 5 reports a $J_{c}$ of $10^{10} \mathrm{~A} \mathrm{~m}^{-2}$ for the grains and $10^{6} \mathrm{~A} \mathrm{~m}^{-2}$ for the transport current at $0 \mathrm{~T}$ and $77 \mathrm{~K}$ ). These results were used for the calculation of the ring magnetization curves. The demagnetization coefficient $\gamma$ in Equation (8) was estimated from the slope of the measured grain magnetization. However, $\alpha$ was used as a fit parameter to reproduce the measured response of the transport current to increased field amplitudes. The values of the parameters used are listed in Table 1.

The calculated transport current for a field amplitude of $3.7 \mathrm{mT}$ (Figure $3 a$ ) is similar to the measured curve (Figure 2a). The grain magnetization shows a little hysteresis. This is a result of the hysteresis of the transport current. As in the unsaturated region the grains just follow the local field which is partly due to the transport current. At an amplitude of $10 \mathrm{mT}$ this hysteresis has a relatively small influence on the grain magnetization, which is virtually linear with the applied field. The corresponding transport current maximum has not shifted compared to the position at an amplitude of $3.7 \mathrm{mT}$. For an amplitude of $20 \mathrm{mT}$ the maximum in the transport current has shifted to the $B_{\mathrm{a}}=0$ axis. Now the grain magnetization has started to saturate. The calculations show a shift of the transport current maxima when the grain magnetization saturates, consistent with measurement.

If we compare the calculated grain magnetization curves (Figure 3) with the measured ones, the hysteresis of the measured curves is larger. This difference can be partly be attributed to the limitations of our model. First, we assume one typical grain size instead of a grain size distribution. The small grains will saturate at lower field amplitudes causing a small hysteresis. Second, we assume a homogeneous magnetization. However, in the ring there will be poorly superconducting regions or even holes. Third, the grains in the ring generally will not be spherical. Next to model limitations the experimental setup plays a major role. In Equation (3) we assume a perfect cancelling of the transport related pickup voltages of the inner and outer pickup set. This does not hold if we look at the signals in detail. The pickup voltages consist of the contributions of different segments, while the transport current hysteresis in each

Table 1 Values of the parameters used in the model calculations

$\begin{array}{|ll|}\alpha=0.3 & \gamma=0.43 \\ J_{0}^{g}=6.0 \times 10^{10} \mathrm{~A} \mathrm{~m}^{-2} & B_{0}^{g}=2 \mathrm{mT} \\ J_{0}^{\mathrm{t}}=1.4 \times 10^{7} \mathrm{~A} \mathrm{~m}^{-2} & B_{0}^{\mathrm{t}}=1 \mathrm{mT}\end{array}$


Interaction between intra-grain currents and transport current in bulk YBaCuO: A.R. Kuper et al.
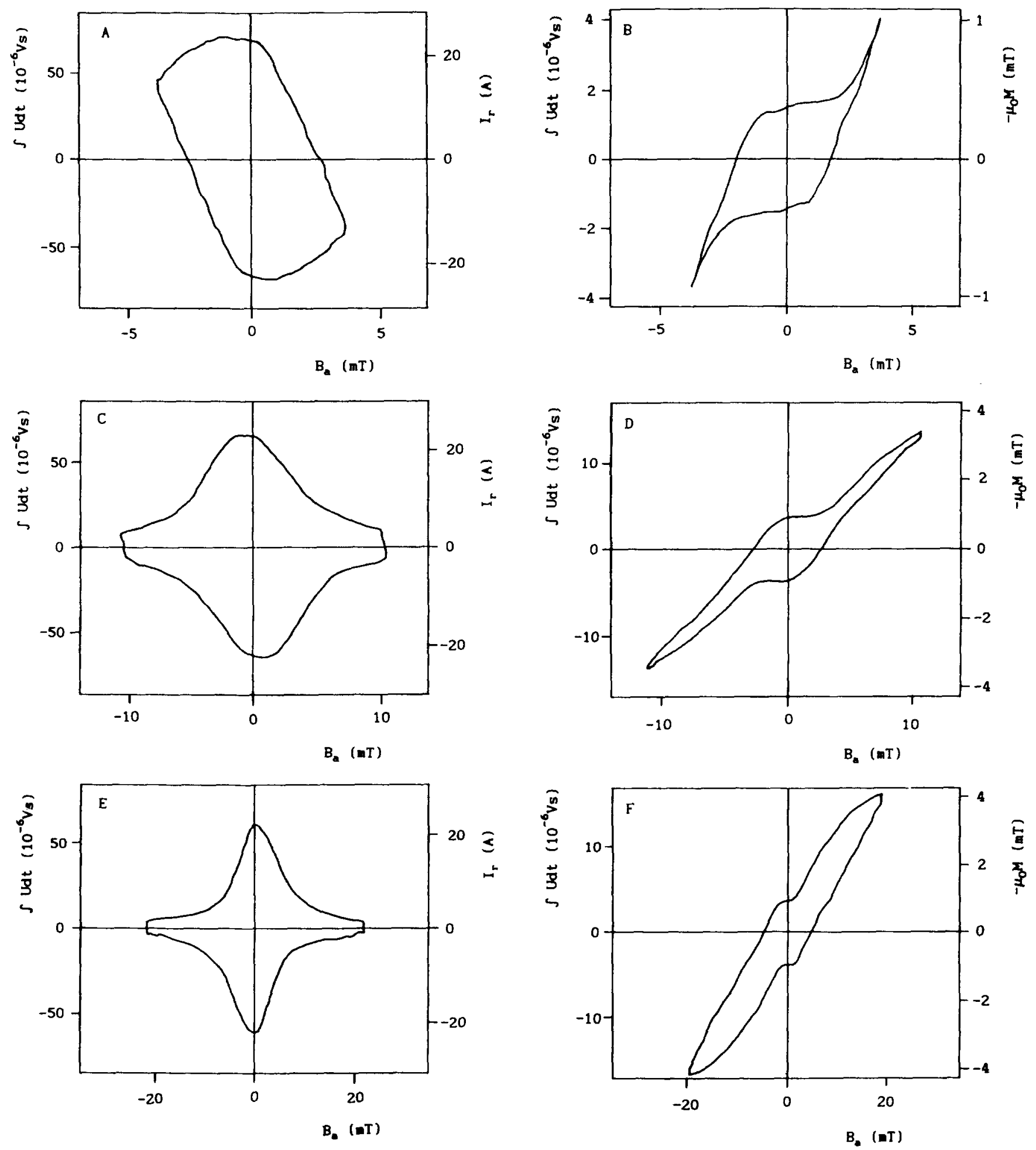

Figure 2 Measured transport current and grain magnetization in a $\mathrm{YBa}_{2} \mathrm{Cu}_{3} \mathrm{O}_{7-\delta}$ ring at $70 \mathrm{~K}$ and increasing field amplitudes

segment as well as the coupling of each segment with the pickup sets is different

$$
\begin{aligned}
& U_{\mathrm{i}}=\sum_{j} M_{\mathrm{i} j} \dot{I}_{j} \\
& U_{\mathrm{u}}=\sum_{j} M_{\mathrm{oij}} \dot{I}_{j}
\end{aligned}
$$

The applied field changes first influence the outer segments. When these saturate the inner segments follow. Therefore, $I_{j}(t)$ has roughly the same shape for all segments but it has shifted in time. We now introduce an average current $I$ and develop the current in segment $j$ with respect to its time shift $\delta t_{j}$ relative to the average current, neglecting the shape differences

$\dot{I}_{\mathrm{j}}=\dot{I}+\ddot{I} \delta t_{j}+O\left(\delta t_{j}^{2}\right)$

With substitution of Equation (12) in the difference 

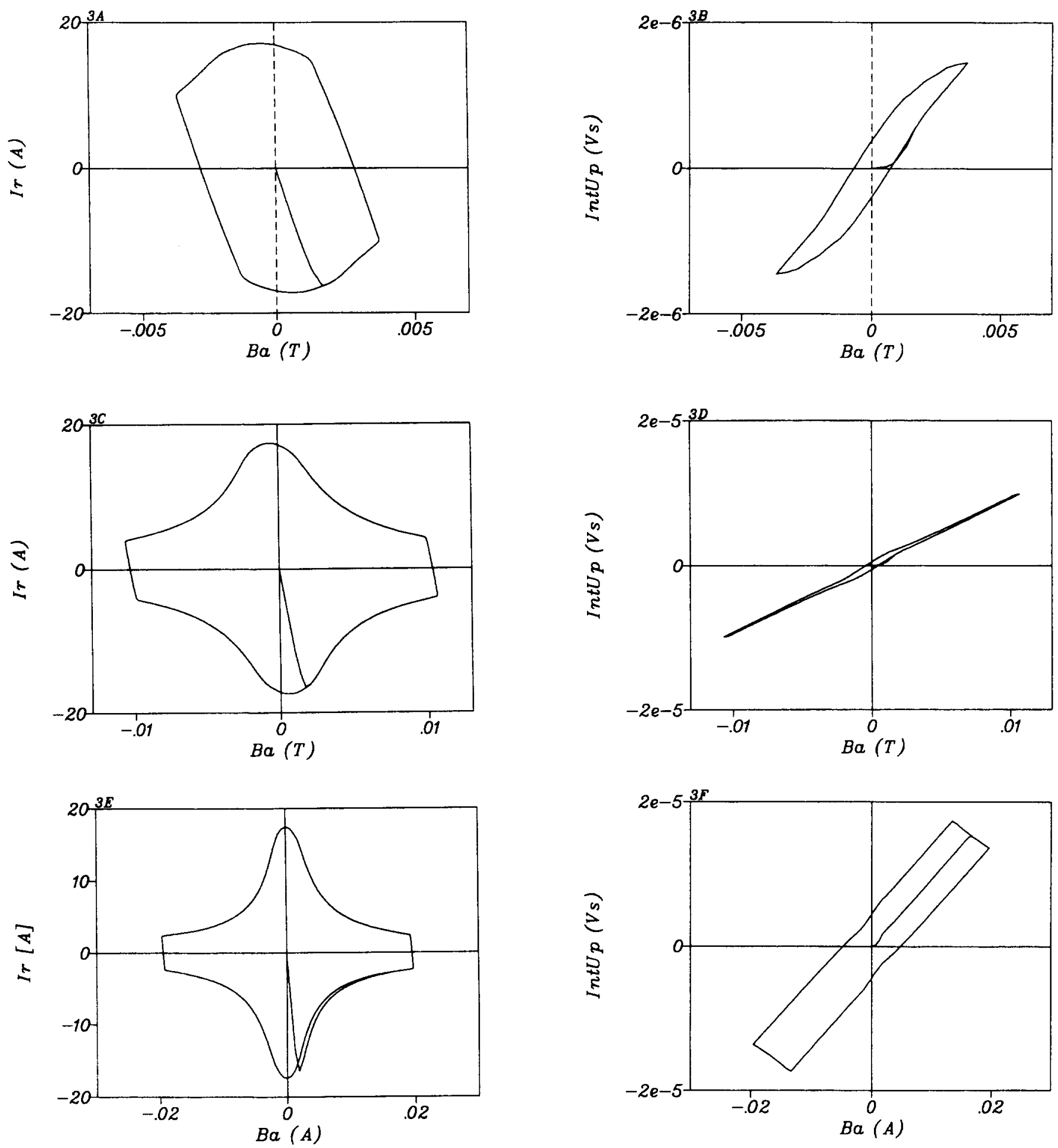

Figure 3 Calculated transport current and grain magnetization at increasing field amplitudes

$U_{\mathrm{i}}-U_{\mathrm{o}}$ we find

$U_{\mathrm{i}}-U_{\mathrm{o}}=\sum_{j} \Delta M_{j} \dot{I}_{j} \cong \dot{I} \sum_{j} \Delta M_{j}+\ddot{I} \sum_{j} \Delta M_{j} \delta t_{j}$

where $U_{\mathrm{i}(o)}$ is the pickup voltage of the inner (outer) pickup set, $M_{\mathrm{i}(\mathrm{o}) j}$, the mutual inductance between segment $j$ and the pickup set, $\dot{I}_{\mathrm{i}}$, the time derivative of the current in segment $j, \delta t_{j}$, the time difference between the average current $I$ and the current in segment $j$ and
$\Delta M_{j}=M_{\mathrm{i} j}-\beta_{\mathrm{t}} M_{\mathrm{o} j}$, the difference in mutual induction of the inner and outer pickup set with the current. The choice of $\beta_{\mathrm{t}}$ in Equation (3b) corresponds to making $\Sigma \Delta M_{j}=0$. However, the two terms in the right-hand side of Equation (12) cannot simultaneously be made zero. There will inevitably be a non-zero contribution of the transport current to the grain magnetization proportional to $\ddot{I}$. For the integrated pickup voltage this is proportional to $\dot{I}$. This is illustrated in Figure 4 . The solid curve is the transport current part of the pickup voltages after the addition as given by Equation ( $3 b)$. The weight 


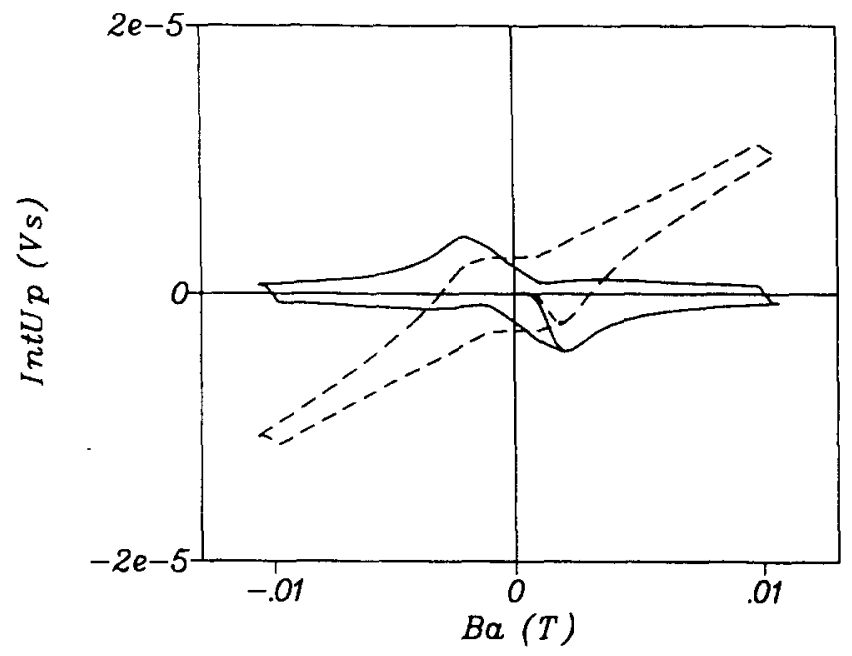

Figure 4 Influence of the imperfect cancelling of the transport current on the measured grain magnetization. The solid curve is the remaining signal after addition of the integrated pickup voltages. If we add this signal to the calculated curve in Figure $3 d$ we get the dashed curve

factor (Equation (4)) is identical to the one used in the measurement. If we add this signal to the calculated grain magnetization in Figure $3 d$ we get the dashed curve. This dashed curve shows a plateau similar to the measured grain magnetization in Figure $2 d$. So the imperfect cancelling of the transport current has a significant influence on the measured grain magnetization. In the same way the plateaux in Figures $2 b$ and $2 f$ can be explained. The grain magnetization related part of the pickup signal is 10 times smaller than the transport current related part. Therefore imperfect cancelling of the grain magnetization has not such a pronounced influence as the imperfect suppressing of the transport current. Furthermore a major part of the grain magnetization is linear in the applied field. This minimizes its contribution after subtraction.

Figure 5 shows the measured transport current for

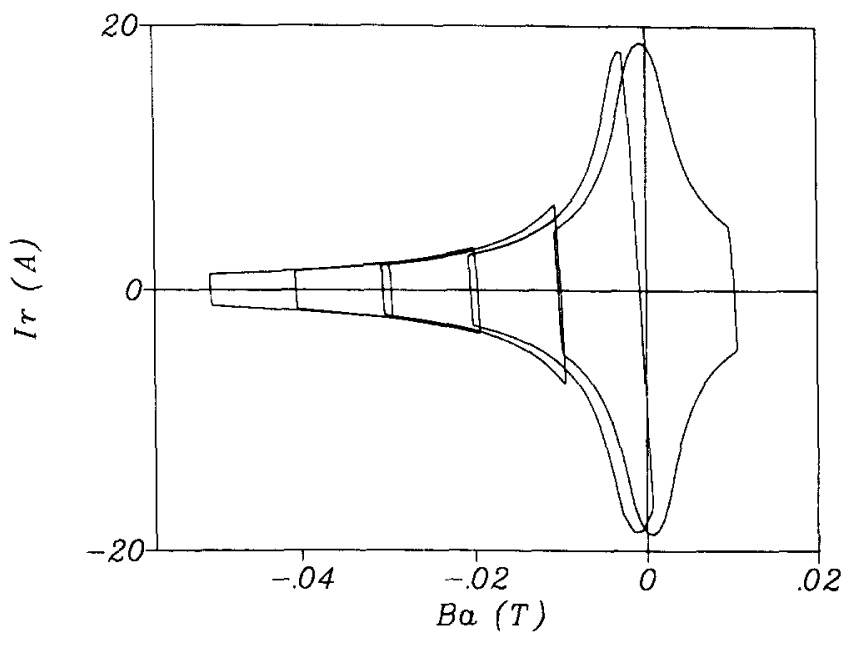

Figure 6 Transport current calculated for different background fields

several d.c. background fields. If there was only a transport current and no grains, the curves with different background fields should connect. Again the hysteresis of the grain magnetization can explain this difference. If we assume saturation of the grains, the grain magnetization at zero d.c. field is as shown in Figures $2 f$ and $3 f$. At minimum $\left|B_{\mathrm{a}}\right|$ the local field is effectively lowered by the grain magnetization induced field. At maximum $\left|B_{\mathrm{a}}\right|$ the local field is increased. These local field changes increase the transport current at minimum $\left|B_{\mathrm{a}}\right|$ and reduce it at maximum $\left|B_{\mathrm{a}}\right|$. Because of the field dependence of the grain magnetization its contribution becomes smaller at higher applied fields and is negligible for high background fields $(>100 \mathrm{mT})$. Then no increase or decrease of the transport current is observed. The calculated transport current corresponding to the measured results in Figure 5 is shown in Figure 6.

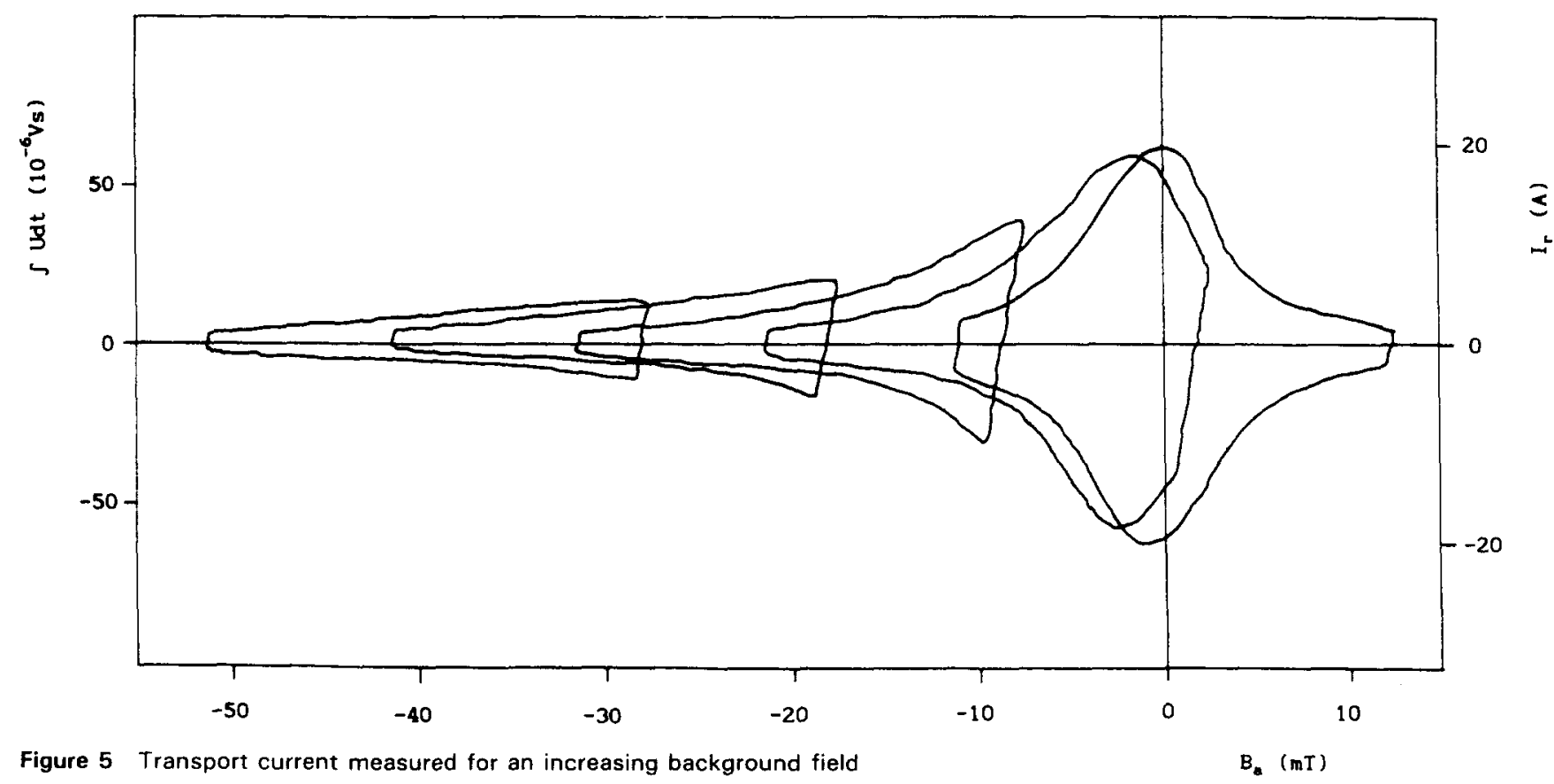




\section{Conclusions}

The transport current and the grain magnetization can be separated in a straightforward way. It makes interpretation of the transport current obtained from magnetization measurements easier, because we can study their mutual influence. As shown, the hysteresis of the grain magnetization has a large effect on the transport current. This can be attributed to the field generated by the grains. A simple model can reproduce the observed transport current and the grain magnetization curves qualitatively. The model can be very helpful in the interpretation of magnetization measurements on inhomogeneous superconductors.

\section{References}

1 Küpfer, H., Apfelstedt, I., Flükiger, R., Keller, C., MeierHirmer, R., Runtsch, B., Turowski, A., Wiech, U. and Wolf, T. Investigation of inter- and intragrain critical currents in high $T_{\mathrm{c}}$ ceramic superconductors Cryogenics (1988) 28 650-660.

2 Eberhardt, F.J., Hibbs, A.D. and Campbell, A.M. Flux trapping and magnetization of hollow superconducting cylinders Cryogenics (1988) $28681-684$

3 Cough, G.E., Colclough, M.S., O'Connor, D.A., Weehofer, F., Alford, N.McN. and Button, T.C. Novel magnetometer and the interpretation of low field magnetic hysteresis of high $T_{\mathrm{c}}$ superconductors Cryogenics (1991) 31 119-127

4 Calzona, V., Cimberle, M.R., Ferdeghini, C., Putti, M. and Siri, A.S. A.C. susceptibility and magnetization of high- $T_{c}$ superconduc- tors: critical state model for the intergranular region Physica $C$ (1989) $157425-430$

5 Evets, J.E. and Glowacki, B.A. Relation of critical current irreversibility to trapped flux and microstructure in polycrystalline $\mathrm{YBa}_{2} \mathrm{Cu}_{3} \mathrm{O}_{7}$ Cryogenics (1988) $28641-649$

6 Bean, C.P. Rev Mod Phys (1964) 3631

7 Kim, Y.B., Hempstead, C.F. and Strand, A.R. Magnetization and critical supercurrents Phys Rev (1963) 129 (2) $528-535$

8 Kuper, A.R., Hemmes, H. and van de Klundert, L.J.M. An AC technique to separate intra grain and inter grain current contributions to the magnetization of high- $T_{\mathrm{c}}$ rings Advances in Cryogenic Engineering Vol 38B, Plenum, New York, USA (1992) $523-529$

9 Fawzi, T.H. and Burke, P.E. The accurate computation of self and mutual inductances of circular coils IEEE Trans Pwr App Syst (1978) 97 (2) $464-468$

10 Blank, D.H.A., Kruidhof, H. and Flokstra, J. Preparation of $\mathrm{Ba}_{2} \mathrm{Cu}_{3} \mathrm{O}_{7-\delta}$ by citrate synthesis and pyrolysis $J$ Phys $D$ (1988) 21 $226-227$

11 Kuper, A.R., Hoogendam, P., Hemmes, H., van de Klundert, L.J.M. and Kolster, B.H. The influence of additives on the current carrying capacity of bulk $\mathrm{YBa}_{2} \mathrm{Cu}_{3} \mathrm{O}_{7-x}$ Physica $B$ (1990) 1385 $165-166$

12 Frankel, D.J. J App/ Phys (1979) 505402

13 Däumling, M. and Larbalestier, D.C. Critical state in disk-shaped superconductors Phys Rev B (1989) $409350-9353$

14 Hemmes, H., Kuper, A.R. and van de Klundert, L.J.M. Time dependent critical state in discs and rings IEEE Trans Magn (1991) 27 (2) $1069-1071$

15 Chaudhary, P., Koch, R.H., Laibowitz, R.B., McGuire, T.R. and Gambino, R.B. Critical current measurements in epitaxial films of $\mathrm{YBa}_{2} \mathrm{Cu}_{3} \mathrm{O}_{7-x}$ Phys Rev Lett (1987) 58 2684-2686

16 Dinger, T.R., Worthington, T.K., Gallagher, W.J. and Sandstrom, R.L. Direct observation of electronic anisotropy in single crystals Phys Rev Lett (1987) 58 2687-2690 